\title{
Electrophysiological Evaluation of the Modified Ashworth Scale in Assessment of Post Stroke Ankle Plantar Spasticity
}

\author{
Chinmayee Patel ${ }^{1}$, Rajiv Limbasiya ${ }^{2}$, Nensi Vaibhav Gandhi ${ }^{3}$ \\ ${ }^{1}$ Assistant Professor, The Shrimad Rajchandra College of Physiotherapy, Uka Tarsadia University, Maliba \\ Campus, Bardoli, Gujarat, India. \\ ${ }^{2}$ Associate Professor, The Sarvajanik College of Physiotherapy, Veer Narmad South Gujarat University, Surat, \\ Gujarat, India. \\ ${ }^{3}$ Assistant Professor, Parul University, Vadodara, Gujarat, India.
}

Corresponding Author: Chinmayee Patel

\begin{abstract}
Background: Spasticity is a common impairment following upper motor neuron lesions such as stroke. The appropriate measure of muscle spasticity, using validated tools to evaluate the outcome of therapies is important in clinical and research settings.

Objective: To determine the concurrent criterion-related validity of the Modified Ashworth Scale in assessing post stroke Ankle flexor muscle spasticity based on its correlation with Modified Tardieu scale and the H-reflex tests.

Methods: A total of 35 adult stroke participants underwent clinical and electrophysiological assessment of the ankle flexor muscle spasticity on the affected side. The primary outcome measures were: MMAS grade, R2-R1 of the MTS; and the H-reflex indices of H-max/M-max ratio.

Results: Correlations tests revealed the correlation between the MMAS and MTS but did not reveal significant associations between the MMAS and the H-reflex tests.

Conclusions: This study suggests that the MMAS may not be a valid tool to evaluate the ankle flexor muscle spasticity in these stroke participants.
\end{abstract}

Keywords: Stroke, spasticity, Modified Ashworth Scale, Tardieu Scale, H-reflex, criterion validity.

\section{INTRODUCTION}

In poor countries, death of stroke patients rates in middle aged people (3065years) are higher in large countries. ${ }^{(1)(2)}$

Damage to the pyramidal tract and its fibers gives rise to the upper motor neuron syndrome including positive and negative features ${ }^{(3)}$. The exact influence of spasticity on motor impairments and activity limitations in stroke participants is difficult to assess because the degree of spasticity may change according to the position of the subject and the task being performed. (4)(5) Clinically, the ankle is often affected post stroke, with $60 \%$ of stroke survivors discharged from in-patient rehabilitation requiring an ankle orthotic and 34\% of stroke survivors developing ankle contracture (6). The measurements of spasticity cover the overall assessment process so it is important to evaluating the reliability and validity of already existing clinical assessment Scales (7) (8). Selecting the proper treatment strategy and goals for the management of post stroke spasticity was result in favorable functional outcomes. Because functional recovery is not depends only on muscle tone but also on strength, co-ordination, endurance and sensation (9). The MTS determines the passive range of movement at different movement velocities, with the relative difference between a slow 
and a fast velocity passive stretch determining the dynamic component of the muscle contracture ${ }^{(10)}$. With the MTS, two resulting joint angles are measured by gonio-meter which include the R1 angle which is the 'angle of catch' after a fast velocity stretch and the $\mathrm{R} 2$ angle defined as the passive joint range of movement following a slow velocity stretch ${ }^{(11)}$. The R2-R1 value indicates the level of dynamic component of spasticity in the muscle. A larger difference between R1 and R2 means large dynamic component, whereas a small difference between R1 and R2 means static contracture in the muscle ${ }^{(12)}$. The MTS uses standardized procedures to measure quality of muscle reaction at the following specified three velocities that can be applied to the muscle. ${ }^{(13)}$ Electro diagnostic studies have contributed to the knowledge about neuronal circuits in the spinal cord but, unfortunately, the results correlate poorly with clinical severity. Kinesiology electromyography studies, measuring changes in muscle function, have shown that the following factors contribute to the impairment of muscle function: increase in stretch reflex activity, inappropriate muscle activation and severity of paresis ${ }^{(14-16)}$.

The Hoffmann reflex ( $\mathrm{H}$ reflex) is one of the electrical analogue of the monosynaptic stretch reflex. The $\mathrm{H}$ $\max / \mathrm{M}-\max$ ratio and latency are conventional indicators of the H-reflex, and are used to study muscle spasticity objectively. The H-reflex latency is usually reduced and the $\mathrm{H}$-max/M-max ratio is increased in stroke participants with muscle spasticity. In stroke participants with spasticity, the hyper excitability of the stretch reflex is neurophysiologic ally characterized by an increase of the ' $\mathrm{H}$ $\max / \mathrm{M}$ max' ratio. ${ }^{(17)}$

\section{METHODOLOGY}

Study Design: This is the Cross sectional study to evaluate the correlation of the Modified Ashworth Scale with the Modified Tardieu Scale and the H-reflex.

Sample Size: 35 subjects
Study Setting: Sarvajanik Physiotherapy College, Surat.

Type Of Population: Acute and sub acute stroke subjects.

Study Duration: March 2015 to February 2016.

Inclusion Criteria: First episode of stroke, History of stroke between 1 to 12 months, Age: $30-65$ years, Ability to understand and follow instruction.

Exclusion Criteria: Fixed contracture at ankle, Knee and ankle pain, Taking antispastic drugs, Contraindication of passive movements at ankle joint Lower limb radiculopathy.

Tools and Materials: room setting, plinth and pillow, pen, paper, record sheet for hreflex: EMG NCS unit, ground electrode, disc electrodes, stimulator electrode box, aqua sonic gel. For Modified Tardieu scale: pen, paper, standard goniometer.

Outcome measures: 1) Modified Modified Ashworth Scale, 2) Modified Tardieu Scale, 3) The H-slp / M-slp ,H-max / M-max ratio. Procedure: A total 35 subjects who were found to be falling under the category of acute and sub acute stroke and who were found to be satisfying all the inclusion criteria were included. A detailed explanation regarding the complete procedure was done for each subject and as a formality towards their willingness to be a part of this study: They were asked to sign written consent. After taking written consent, all patients was be asked to rest on plinth or chair or on the bed with shoes removed for 5 minutes and remain comfortable and relaxed. After getting all these information and giving proper positions as mentioned below the clinical tests like the modified Ashworth scale and Modified Tardieu scale was be performed first and then H-reflex. Tests are performed in quiet and proper ventilated room with electrically shielded and earth grounded for h-reflex test. Planter flexor spasticity of the affected side can be assessed by using Modified Ashworth and Modified Tardieu scale and the neuro physiological data was also be collected from affected side. 
The Modified Ashworth Scale: For Gastronomies: Start position: Supine, ankle plantar flexed, hip in neutral rotation and flexion.

Movement: Dorsi-flex the patient's ankles from maximum possible plantar flexion to maximum possible dorsi-flexion not more than three consecutive times and rate the muscle tone. For Soleus: Start position: Supine, ankle plantar flexed, hip in neutral rotation and flexion and with the knee flexed to $\sim 15^{\circ}$.

Movement: Dorsiflex the patient's ankle from maximum possible plantar flexion to maximum possible dorsi-flexion. The therapist was score muscle spasticity from 0-4.

Modified Tardiue Scale: The test procedure for the ankle plantar flexors was as follows:

Starting position: All subjects were placed in supine position with ankle joint out of the couch with knee in extension. Marked one point at the lateral aspect of lateral malleolus and another point were marked on the head of fibula for reference by a removable marker. The first line was drawn joining these two points and the second line was drawn parallel to the lateral aspect of the fifth metatarsal. After positioning of the axis over the lateral malleolus with stabilizing arm along the first line and movable arm along the second line, the gonio-meter was fixed by two Velcro over mid-foot and above ankle. After that moved the ankle joint first with a very slowstretching velocity (V1) and fast stretching velocity (V3), from plantar flexion towards dorsi-flexion. The joint was moved first with a very slow-stretching velocity (V1) from plantar flexion towards dorsi flexion to measure the PROM by counting. During this maneuver, the catch was noted by the rater and $\mathrm{R} 2$ was documented by the observer. Quality of muscle reaction (MTS scores) ranging from 0 to 4 grades were rated by the rater at the stretching velocity of V2. The angle of muscle reaction was measured at the point of resistance to the fastest stretching velocity V2 by counting 1 ,
2, 3 onwards. The angle of catch (R1) was noted by observer. Throughout the procedure, the rater was blinded by covering the goniometer with opaque tape and documented all values. The grading of spasticity of the ankle flexor muscles is according to the annurix -5 .

Measurement of H-reflex: Position: Patient should be in semi reclining comfortable or lie in prone position with leg and thigh firmly supported. The feet should be hanging freely with dorsum at the right angle to tibia. Placement of electrodes for the H-reflex Stimulation: Site of stimulation for the H-reflex A square wave pulse of $1 \mathrm{~ms}$ duration is used for preferential stimulation of large sensory fibers. The cathode is kept proximal to anode to avoid anodal block. Stimulus frequency should not exceed first in $5 \mathrm{sec}$ to exclude any effect of prior stimulus. The stimuli are adjusted so as to evoke maximum $\mathrm{H}$ response amplitude. At this strength a small $\mathrm{M}$ response may help in monitoring the strength of stimulation. At least $5 \mathrm{H}$ responses should be studied for analysis. By increasing the stimulus strength to supra-maximal, maximum $\mathrm{M}$ response can be recorded and $3 \mathrm{M}$ responses are measured for analysis. Recording and Measurement: The H-reflex and M-wave was be obtained by Neurostim diagnosis EMG NCS UNIT machine. The band pass filter is set at $5 \mathrm{~Hz}$ to $3 \mathrm{~Hz}$, sweep rate at 5 $\mathrm{ms} / \mathrm{div}$ and sensitivity at 500 uuv to $2 \mathrm{mv} / \mathrm{div}$ was be used. Electrical pulses $1 \mathrm{~ms}$ in duration were repeat at every $5 \mathrm{sec}$. The output of which ranged from 0-100 uuA. The electric resistance between two electrodes was be less than $10 \mathrm{kHz}$. The latency of $\mathrm{H}$ reflex is measured from the stimulus artifact to the first deflection from the baseline. The amplitude is measured from base to peak of the negative phase. To build the recruitment curves of H-reflex and M-waves, five pulses was be delivered sequentially and the mean amplitude of evoked H-reflexes and the M-responses was be calculated.

The amplitudes were be measured peak to peak. Mean amplitudes was be 
normalized according to the following formula: Normalized amplitude $=$ (Mean amplitude of five waves)/M max $* 100$ Stimulus Intensities was be presented as the ratio of the threshold intensity of the $\mathrm{M}$ wave $\left(\mathrm{M}^{\mathrm{th}}\right)$.All data measured at stimulus intensities less than the threshold of the Mwave was be used to determine the H-slp. Data from $\mathbf{M}^{\text {th }}$ up to maximum amplitude of M-wave was determine the M-slp. Equation of simple linear regression was be determined, and the slope of this line was be considered as $\mathrm{H}$-slp or M-slp. Maximum mean amplitude of the H-reflex and maximum mean amplitude of the $\mathrm{M}$-wave was regarded as H-max and M-max, respectively. The H-max relative to M-max is H-max/M-max ratio. The H-reflex latency was calculated from stimulus wave to the onset of first deflection of the H-wave, and the amplitude of recorded reflexes and waves was be measured.

\section{RESULT}

Descriptive statistics including mean, standard deviation for age and post stroke duration were analyzed. Frequency percentages of gender and side affection were analyzed. Concurrent validity was examined by correlating the average score of the MMAS with the MTS and H-reflex by using the Spearman's Rank correlation coefficient (Rho).The obtained result were considered significant if the value of $\mathrm{p}<0.05$.

Table 1: Demographic data of Participants $(n=35)$

\begin{tabular}{|l|l|}
\hline & $($ Mean \pm SD) \\
\hline Age & $51.49 \pm 9.153$ \\
\hline Post stroke duration (in months) & $9.09 \pm 2.769$ \\
\hline
\end{tabular}

Gender distribution among the stroke participants: there were $77 \%$ of male and $23 \%$ of female. Affected side distribution among the stroke participants: there were $80 \%$ of right sided and $20 \%$ of left sided.

Table:2: Participants' descriptive result of MMAS and MTS $(\mathbf{n = 3 5})$

\begin{tabular}{|l|l|}
\hline Variables & (Mean \pm SD) \\
\hline MMAS Grades & $1.77 \pm .426$ \\
\hline R-1 Value & $12.31 \pm 5.212$ \\
\hline R-2 Value & $16.69 \pm 2.816$ \\
\hline R1-R2 value & $4.37 \pm 4.38$ \\
\hline MTS Grade & 1.71 .458 \\
\hline
\end{tabular}

\begin{tabular}{|c|c|c|c|c|c|c|}
\hline \multirow[t]{3}{*}{ Spearman's Rho } & & MMAS Grade & R1 value & R2 value & R2 - R1 value & MTS Grade \\
\hline & Correlation coefficient & 1.000 & $-.339 *$ & $-.618 * *$ & $-.341 *$ & $.861^{* *}$ \\
\hline & sig. (2-tailed) & . & .046 & .000 & .045 & .000 \\
\hline
\end{tabular}

Table 4: Participants' descriptive result of MMAS and H-reflex $(n=35)$

\begin{tabular}{|l|l|}
\hline Variables & (Mean \pm SD) \\
\hline MMAS grade & $1.77 \pm .426$ \\
\hline M Latency & $6.28 \pm .008$ \\
\hline H latency & $30.87 \pm 2.010$ \\
\hline H amplitude & $2.66 \pm 2.817$ \\
\hline H-max / M-max ratio & $4.91 \pm .319$ \\
\hline
\end{tabular}

Table -5: Spearman's Rho correlation of the MMAS and the H-reflex:

\begin{tabular}{|l|l|l|l|l|l|l|}
\hline \multirow{3}{*}{ Spearman's Rho } & MMAS Grade & M Latency & H Latency & H amplitude & H-max/M-max Ratio \\
\cline { 2 - 7 } & Correlation coefficient & 1.000 & -.093 & -.039 & $.364 *$ & -.039 \\
\cline { 2 - 6 } & sig.(2-tailed) &. & .594 & .826 & .032 & .826 \\
\hline
\end{tabular}

\section{DISCUSSION}

The participants of the stroke had a lower grade of spasticity were observed in the present study, this may be due to the patient receiving the rehabilitation interventions. So no participants were graded ' 3 ' and ' 4 ' for MMAS. The participants who had MTS grades of ' 3 ' and ' 4 ' were not included in the present study for the calf muscle spasticity. The speed of movement used in this study for measuring spasticity was V3. The velocity used for stretching of muscle in the present study did not appear to be fast enough to elicit clonus. The reason for absence of grades ' 3 ' and ' 4 ' in the assigned grades of MTS may be that the clonus is an independent phenomenon. The most of participants were found for 
assigned grade '2' of MTS. This may indicate that the MTS may provide more reliable measurements with spastic muscles. Stronger correlations might have been demonstrated if the all grades MTS of spasticity had been tested, or if large numbers of stroke participants with higher MMAS grades had been included. Muscle spasticity is usually examined by testing reflex excitability in electrophysiological studies by H-reflex. The H-max / M-max ratio and latency are conventional indicators of the H-reflex, and are used to study muscle spasticity objectively. The $\mathrm{H}$ latency is usually reduced and the H-max / M-max ratio is increased in patients with muscle spasticity. The H-slp / M-slp ratio is a better indicator for evaluating the alpha motor neuron excitability. The $\mathrm{H}$-slp, more sensitive than the H-max / M-max ratio, provides more information about the recruitment threshold. But in this study because of instrumental error, the H-slp / Mslp couldn't obtain. So may be the absence of H-slp / M-slp measurement, the result shows negative correlation between the MMAS and H-reflex. In participants with spasticity H-max / M-max ratio is increased due to the lack of inhibition associated with muscle relaxation. The discrepancy between the previous and this studies may be because of they used the H-slp / M-slp ratio which has been more sensitive measures at assessing excitability of the motor neurons because it excludes alteration in peripheral factors from influencing $\mathrm{H}$-reflex amplitude.

The gender and duration of stroke condition will also affecting the result of this study. The results of the present study are not consistent with previously reported findings on correlation of MMAS with $\mathrm{H}$ reflex in stroke participants with wrist flexor spasticity. The reason may be the large number of patients recruited for this study.

The results of the present study are not consistent with previously reported findings on relationship of the MMAS with the H-reflex.

\section{CONCLUSION}

The result of this study reveals a significant positive correlation between the MMAS and the MTS. But failed to reveal a significant correlation between the MMAS and the H-reflex tests. The lack of significant correlations between the MMAS and the H-reflex tests indicate that electrophysiological, the MMAS may not be a valid measurement tool of ankle flexor muscle spasticity in stroke participants.

Ethical Clearance: Obtained from the institutional committee of ethics of the Sarvajanik College of Physiotherapy.

\section{Acknowledgement: None}

\section{Conflict of Interest: None}

\section{Source of Funding: Self}

\section{REFERENCES}

1. Strong Ket al. Preventing stroke: saving lives around the World. The Lancet Neurology.2007; 6: 182-187.

2. Mather $\mathrm{C}$ et al. Projections of global mortality and burden. PLoS Medicine. 2006 November; 3(11).

3. O' Dwyer NJ et al. Spasticity and muscle contracture following stroke. Brain. 1996; 119.

4. Watkins CL et al. Prevalence of spasticity post stroke. Clin Rehabil. 2002; 16: 515522.

5. N.SMANIA e al. Rehabilitation procedures in the spasticity. Eur $\mathrm{j}$ phys rehabil med 2010; 46.

6. Wasay $M$ et al. Stroke in South Asian countries. Nat. Rev. Neurol. 2014; 10.

7. Ashworth B. Preliminary trial of Carisoprodol in multiple sclerosis. Practitioner. 1964;192:540-42.

8. Bohannon $\mathrm{R}$ Wet al. Intera-rater reliability of a modified Ashworth scale of muscle spasticity. Phys The. 1987; 67(2).

9. Bipin B Bhakta. Management of Spasticity in Stroke. British Medical Bulletin 2005, 56.

10. Morris S. Ashworth and Tardieu scales: Their clinical relevance for measuring spasticity in adult and pediatric neurological populations. Physical Therapy Reviews 2002; 7. 
11. Ansari, N.N.et al. Clinical assessment of ankle plantar flexor spasticity in adult patients after stroke: Inter- and intra raterreliability of the Modified Tardieu Scale. Brain Injury,2013.605-612.

12. Abolhasani, H.et al. Compare the validity of the Modified Modified Ashworth Scale and the Modified Tardieu Scale in the assessment of wrist flexor spasticity in patients with Stroke: Protocol for a neurophysiological study.2012 BMJ Open.

13. Fellows' SJ et al. Agonist and antagonist EMG activation during isometric torque development at the elbow in spastic hemi paresis. Electroencephalogry Clinical Neurophysiology 1994; 93:106-12.

14. AF Thilmann et al. The time-course of bilateral changes in the reflex excitability of relaxed triceps surae muscle in human hemiparetic spasticity. J Neurol 1991; 238.(5):293-8.

15. Thilmann A, Fellows SJ. The mechanism of spastic muscle hypertonus. Brain. 1991; 114:233-44.

16. Bishop B et al. A quantitative assessment of gamma-motoneuron contribution to the Achilles tendon reflex in normal subjects. Archives of Physical Medicine \& Rehabilitation 1968; 49:145-154.

17. Nielsen Jet al. H-reflex in spastic spinal cord injured patients. Experimental Brain Research 1993; 97:173-17.

How to cite this article: Patel C, Limbasiya R, Gandhi NV. Electrophysiological evaluation of the modified ashworth scale in assessment of post stroke ankle plantar spasticity. Int $J$ Health Sci Res. 2021; 11(8): 102-107. DOI: https://doi. org/10.52403/ijhsr.20210815 